\title{
The evaluation of the geoid-quasigeoid separation and consequences for its implementation
}

\author{
Joachim Schwabe ${ }^{1,2} \cdot$ Martin Horwath $^{1}$. \\ Mirko Scheinert ${ }^{1}$
}

Received: 23 April 2015/Accepted: 21 July 2015/Published online: 30 July 2015

(C) Akadémiai Kiadó 2015

\begin{abstract}
The realization of precise height systems demands to assess the effect and necessity of approximations made to the pure theory. In this context, the formulas for the geoid-quasigeoid separation as presented by Flury and Rummel (J Geod 83:829-847, 2009) and further discussed by Sjöberg (J Geod 84:699-702, 2010) are reinterpreted. Starting from the fully topographically reduced gravity disturbance, a modification of the strict formulation of the downward continuation and the indirect effect according to Sjöberg (2010) is given. In practice any implementation of the formula requires approximations in order to realize the downward continuation of gravity along the plumbline with the help of density assumptions and a topography model. The significance of the individual contributors to a refined approximation, taking into account the indirect effect and the firstorder gravity gradient, is elaborated in a numerical simulation for the example of the Himalaya region. Special focus is given on the sensitivity and convergency of the topography-induced terms with respect to the integration radius.
\end{abstract}

Keywords Geoid · Quasigeoid · Height anomaly · Indirect effect

\section{Introduction}

The practical evaluation of the geoid-quasigeoid separation (GQS) term is a subject of ongoing discussion (e.g. Flury and Rummel 2009; Sjöberg 2010; Vaníček et al. 2012). We believe that its practical importance will increase in the future. More and more, national height reference networks are based on precise geoid or quasigeoid models so that, except for base networks of fundamental stations, spirit leveling can be replaced by satellite

Joachim Schwabe

joachim.schwabe@tu-dresden.de; joachim.schwabe@bkg.bund.de

Institut für Planetare Geodäsie, Technische Universität Dresden, 01062 Dresden, Germany

2 Present Address: Bundesamt für Kartographie und Geodäsie, Karl-Rothe-Straße 10-14, 04105 Leipzig, Germany 
positioning techniques in daily use. Consequently, precise knowledge of the GQS is a prerequisite for accurate conversion between orthometric and normal heights, and thus for the envisaged unification of national height systems. In this context, the GQS may also be relevant in remote areas where spirit leveling is not feasible but where precise height information is a requirement for other non-geodetic applications, e.g., water construction, environmental monitoring, etc.

The well-known formula for the GQS according to Heiskanen and Moritz (1967)

$$
N=\zeta+\frac{\bar{g}-\bar{\gamma}}{\bar{\gamma}} H
$$

allows to determine the geoid from ground-based gravity anomalies adding some corrections. $N$ is the geoid height, $\zeta$ is the (surface) height anomaly, $\bar{g}$ is the mean gravity between the geoid and the surface, $\bar{\gamma}$ is the mean normal gravity between the ellipsoid and the telluroid, and $H$ is the surface height w.r.t. the geoid. This relation directly follows from the basic definitions of the Helmert orthometric height $H$

$$
C=H \bar{g}=\int_{H^{\prime}=0}^{H} g \mathrm{~d} H^{\prime} \doteq \int_{h^{\prime}=N}^{h} g \mathrm{~d} h^{\prime}
$$

and of Molodenski's normal height $H^{N}$

$$
C=H^{N} \bar{\gamma}=\int_{H^{N^{\prime}}=0}^{H^{N}} \gamma \mathrm{d} H^{N^{\prime}} \doteq \int_{h^{\prime}=0}^{h-\zeta} \gamma \mathrm{d} h^{\prime},
$$

respectively. $C$ is the geopotential height of the surface point with respect to the geoid and $h$ is the corresponding ellipsoidal height. $h^{\prime}$ is the ellipsoidal height of the integration point running along the plumbline.

In common practice, Eq. 1 is approximated by the expression

$$
\bar{g}-\bar{\gamma} \approx \Delta g^{\mathrm{B}},
$$

where $\Delta g^{\mathrm{B}}$ is introduced as the Bouguer gravity anomaly. However, various definitions of Bouguer anomalies are employed: both the refined (complete, i.e., including terrain effects) or the simple (incomplete, i.e., without considering terrain effects) Bouguer anomaly may be evaluated in either planar or spherical representation. So far, no common understanding has been achieved which of these types of Bouguer anomalies is to be used in the context of Eq. 4. For example, in the calculation service provided by the International Center for Global Earth Models (ICGEM) the plate reduction is considered (Barthelmes 2009). This approach is commonly associated with the Poincaré-Prey gradient (Heiskanen and Moritz 1967) which is used as a proxy for the actual gravity gradient inside the topographic masses in the definition of Helmert orthometric heights. In view of the growing requirements of accurate height systems and the recent developments in the field of gravity forward modeling from topography models (e.g. Hirt 2013), this approximation may be too inaccurate not only in mountaineous regions (e.g. Sjöberg 2010).

Flury and Rummel (2009) presented a refined approximative formula, taking the indirect effect into account. However, they eventually omit even the vertical gradient of $\Delta g^{\mathrm{B}}$ inside the topography. Sjöberg (2010) discussed this aspect and derived another strict formula, thereby in detail emphasizing the theoretical differences between the Bouguer 
gravity anomaly in the strict sense and so-called non-topographic quantities which lead to a slightly more complex derivation. In conclusion, both formulas are expected to yield the same numerical results at centimeter level almost anywhere on Earth, at least from the theoretical point of view (Sjöberg 2010; Flury and Rummel 2011).

However, the latter authors do not finally provide estimates for a larger area regarding the terms related to the downward continuation and the indirect effect of topography. Therefore, the aim of this paper is not to give a complete review of all the aspects and theoretical facets of the GQS. Instead, the motivation of the study is to investigate the actual magnitude and spatial pattern of the terms that have been often neglected previously. Special focus is given to the role of the integration radius of the topographic reduction terms. However, we do not deal with the remaining problem of the unknown density structure inside the topography which will always remain the major error source in the computation of the GQS.

To begin with, we present another strict (theoretical) formulation which is derived in Sect. 2. For practical evaluation a first-order approximation of the topographically reduced gravity is considered in Sect. 2.2. This modified practical approach is then compared with Eq. 4 and the proposed refinements of Flury and Rummel (2009) and Sjöberg (2010). In this context, we also discuss the GQS as a realization of a topographic reduction scheme without condensation. The practical implementation of the topographic effects is discussed in Sect. 2.3. Based on simulated gravity data and a topography model, numerical results in the Himalaya region are derived and discussed in Sect. 3. Finally, conclusions are drawn in Sect. 4.

\section{Approach}

As a main difference in comparison to Sjöberg (2010) we start from the gravity disturbance $\delta g$ rather than from the gravity anomaly $\Delta g$. Previously, gravity anomalies have been used almost exclusively. However, the relevance of gravity disturbances is increasing. Due to the availability of ellipsoidal heights from satellite positioning new gravity data are usually gravity disturbances. This is also true for airborne gravity campaigns of formerly inaccessible areas, such as mountain regions, deserts, or rain forests. Gravity anomalies may be converted to gravity disturbances by the relation

$$
\delta g-\Delta g=-\frac{\partial \gamma}{\partial h} \cdot \zeta \approx+0.3086 \frac{\mathrm{mGal}}{\mathrm{m}} \cdot \zeta,
$$

if the height anomaly $\zeta$ is given. In the most common case, the GQS is to be applied to $\zeta$, anyway. Looking at any specific region of the Earth, errors of $\zeta$ on the level of $0.1 \mathrm{~m}$ (or even $1 \mathrm{~m}$ ) propagate to errors of the conversion Eq. 5 on the level of $0.03 \mathrm{mGal}$ (or $0.3 \mathrm{mGal}$, resp.) which is unlikely to exceed the accuracy of the corresponding terrestrial gravity datasets in the very same area: The more accurately terrestrial gravity is known, the more accurately the height anomaly can be computed, and the more accurately $\Delta g$ may be converted to $\delta g$, accordingly.

\subsection{Modified strict formula based on the gravity disturbance and first-order approximation}

Bruns' formula

$$
T_{P}=\zeta \cdot \gamma_{Q}, \quad T_{g}=N \cdot \gamma_{0}
$$


is taken for the disturbing potential at the surface $\left(T_{P}\right)$ and at the geoid $\left(T_{g}\right)$, resp., where $\zeta$ is the (surface) height anomaly, $N$ is the geoid height, and $\gamma_{Q}$ and $\gamma_{0}$ are the normal gravity at the telluroid and at the ellipsoid, respectively. Using the relation

$$
\delta g=-\frac{\partial T}{\partial h},
$$

the transition from $T_{P}$ to $T_{g}$ can be expressed by

$$
T_{g}-T_{P}=\int_{h^{\prime}=N}^{h_{P}} \delta g \mathrm{~d} h^{\prime}
$$

Inserting Eq. 8 into Eq. 6, one obtains after some conversions

$$
N-\zeta=\zeta\left(\frac{\gamma_{Q}}{\gamma_{0}}-1\right)+\frac{1}{\gamma_{0}} \int_{h^{\prime}=N}^{h_{P}} \delta g \mathrm{~d} h^{\prime}
$$

A similar decomposition was already found by Sjöberg (2006), Eqs. 3-5. Here, we use this relation explicitly.

We may decompose $\delta g$ into the non-topographic gravity disturbance $\delta g^{\text {nt }}$ and the attraction of the topography $A^{\mathrm{T}}$ (Sjöberg 2010)

$$
\begin{gathered}
\delta g=\delta g^{\mathrm{nt}}+A^{\mathrm{T}}, \\
A^{\mathrm{T}}=-\frac{\partial V^{\mathrm{T}}}{\partial h},
\end{gathered}
$$

where $V^{\mathrm{T}}$ is the corresponding topographic potential. Considering the relation (Flury and Rummel 2009; Sjöberg 2010)

$$
\int_{h^{\prime}=N}^{h_{P}} A^{\mathrm{T}} \mathrm{d} h^{\prime}=V_{g}^{\mathrm{T}}-V_{P}^{\mathrm{T}}
$$

it follows that

$$
\int_{h^{\prime}=N}^{h_{P}} \delta g \mathrm{~d} h^{\prime}=\int_{h^{\prime}=N}^{h_{P}} \delta g^{\mathrm{nt}} \mathrm{d} h^{\prime}+V_{g}^{\mathrm{T}}-V_{P}^{\mathrm{T}} .
$$

In Eq. 13 the total effect of the vertical integration of $\delta g^{\text {nt }}$ may then be formally split into a constituent $K_{\delta g}$ of the surface value $\delta g_{P}^{\text {nt }}$ and a gravimetric correction $K_{\Delta \delta g}$ taking into account the vertical gradient of $\delta g^{\text {nt }}$.

$$
\int_{h^{\prime}=N}^{h_{P}} \delta g \mathrm{~d} h^{\prime}=\delta g_{P}^{\mathrm{nt}} \cdot H_{P}+\int_{h^{\prime}=N}^{h_{P}}\left(\delta g^{\mathrm{nt}}-\delta g_{P}^{\mathrm{nt}}\right) \mathrm{d} h^{\prime}+V_{g}^{\mathrm{T}}-V_{P}^{\mathrm{T}} .
$$

Altogether, we find the new expression for the GQS

$$
N-\zeta=K_{\delta g}+K_{\Delta \delta g}+K_{\text {ind }}+K_{\gamma},
$$




$$
\begin{gathered}
\text { where } \\
K_{\delta g}=\frac{\delta g_{P}^{\mathrm{nt}}}{\gamma_{0}} H_{P}, \\
K_{\Delta \delta g}=\frac{1}{\gamma_{0}} \int_{h^{\prime}=N}^{h_{P}}\left(\delta g^{\mathrm{nt}}-\delta g_{P}^{\mathrm{nt}}\right) \mathrm{d} h^{\prime}, \\
K_{\text {ind }}=\frac{V_{g}^{\mathrm{T}}-V_{P}^{\mathrm{T}}}{\gamma_{0}}, \quad \text { and } \\
K_{\gamma}=\zeta\left(\frac{\gamma_{Q}}{\gamma_{0}}-1\right) .
\end{gathered}
$$

In particular, the term $K_{\text {ind }}$ (Eq. 15d) can be interpreted as the indirect effect on the potential caused by the complete topographic reduction (see also Sect. 2.2).

Assuming a constant vertical gradient of $\delta g^{\text {nt }}$, i.e., applying a first-order Taylor expansion of $\delta g^{\text {nt }}$ in the surface point $P$, the effect of $K_{\Delta \delta g}$ (Eq. 15c) may be approximated to the first order by (see e.g. Sjöberg 2010, Eq. 20)

$$
K_{\Delta \delta g} \approx-\frac{H_{P}^{2}}{2 \gamma_{0}}\left(\frac{\partial \delta g^{\mathrm{nt}}}{\partial h}\right)_{P} .
$$

\subsection{Comparison with existing formulations}

Any practical evaluation of Eq. 1 to infer the geoid from the quasigeoid requires some density hypothesis in order to approximate the unknown actual gravity inside the topography. A number of papers have presented different approaches to derive practical formulas (e.g. Sjöberg 1995; Rapp 1997; Tenzer et al. 2005, 2006; Vaníček et al. 2012) to mention only a few. It appears that, when dealing with the GQS, most authors use formulations that closely follow their preferred approach of geoid determation. One possible perspective is to break down the procedure in the following three main steps: (1) removal of a topographic reduction in terms of gravity from the in-situ data, (2) integration along the plumbline and (3) subsequent restoration of the indirect effect of the topography in terms of the potential. All these contributions can be expressed by means of corrections. In this view, Eq. 4 may be interpreted already as a zero-order realization of this procedure where the vertical gradient of the Bouguer anomaly in step 2 (corresponding to Eq. 15c) and the indirect effect (step 3, corresponding to Eq. 15d) are neglected.

The expressions of Eq. 15a-15e are general, i.e., in theory any arbitrary but appropriate type of topographic reduction (e.g., with or without considering a condensation scheme) is imaginable at this stage (see also Sect. 2.3) as long as the effects expressed by Eqs. 10 and 12 are applied consistently. For example, if a Helmert condensation scheme of the topography was used in Eq. $10, K_{\text {ind }}$ would in fact resemble the transition from the cogeoid to the geoid. However, for this study we will concentrate on the complete topographic reduction without condensation. In this context, $V_{g}^{\mathrm{T}}-V_{P}^{\mathrm{T}}$ represents the corresponding indirect effect on the potential.

The additional correction $K_{\gamma}$ is the most striking difference between Eq. 15a (this study) and Flury and Rummel (2009), Eq. 24, and Sjöberg (2010), Eq. 10a. As we found in a 
simulation based on EGM2008 height anomalies (Fig. 1, see also Sect. 3) its magnitude does not exceed $2 \mathrm{~cm}$ for most regions of the Earth and is limited to $12 \mathrm{~cm}$ even at the highest mountains. For positive topographic heights its sign is opposite to that of the height anomaly. The term $\gamma_{0} \cdot K_{\gamma}$ may be interpreted as the part of the vertically integrated indirect effect $\zeta \frac{\partial \gamma}{\partial h}$ induced by the vertical change in $\gamma$. It compensates the use of the gravity disturbance instead of the gravity anomaly and of $\gamma_{0}$ instead of $\bar{\gamma}$ in the other components of Eq. 15a, which, apart from taking $\gamma$ at the ellipsoid, then mostly resemble respective terms of the aforementioned existing formulas:

$K_{\delta g}$ (Eq. 15b) is the counterpart to the main constituents of Flury and Rummel (2009), Eq. 24, and Sjöberg (2010), Eq. 10a, i.e., it resembles Eq. 4 of this paper.

As to $K_{\Delta \delta g}$, Eqs. $15 \mathrm{c}$ and 16 are similar to the so-called gravimetric correction $G C$ of Sjöberg (2010), Eqs. 10c and 20.

Likewise, $K_{\text {ind }}$ (Eq. 15d) is similar to the topographic correction TC1 of Sjöberg (2010), Eq. 12b.

So, what is the benefit of the new formulation based on the gravity disturbance if it is equivalent to the already existing ones? It is not anymore necessary to consider the secondary indirect topographic effect on gravity! Considering Eq. 7, the additional term $\frac{V^{\mathrm{T}}}{\gamma} \frac{\partial \gamma}{\partial h}$ in Eq. 10b of Sjöberg (2010) vanishes if Eq. 9 of Sjöberg (2010) is formulated for the nontopographic gravity disturbance $\delta g^{\mathrm{nt}}$. Thus, the secondary effects as discussed by Sjöberg (2010), Sect. 3.1, are avoided at first by using the gravity disturbance instead of the gravity anomaly. In this case, the quantities $T C$ and TC1 [Sjöberg (2010) Eqs. 10b,12b] become identical and differ from TC2 [Sjöberg (2010), Eq. 17a] and $K_{\text {ind }}$ (this study, Eq. 15d) only by the respective normal gravity values. As a convenient side effect, the mean normal gravity along the plumbline does not anymore show up explicitely so that the normal gravity in Eq. 15a may be computed right at the ellipsoid.

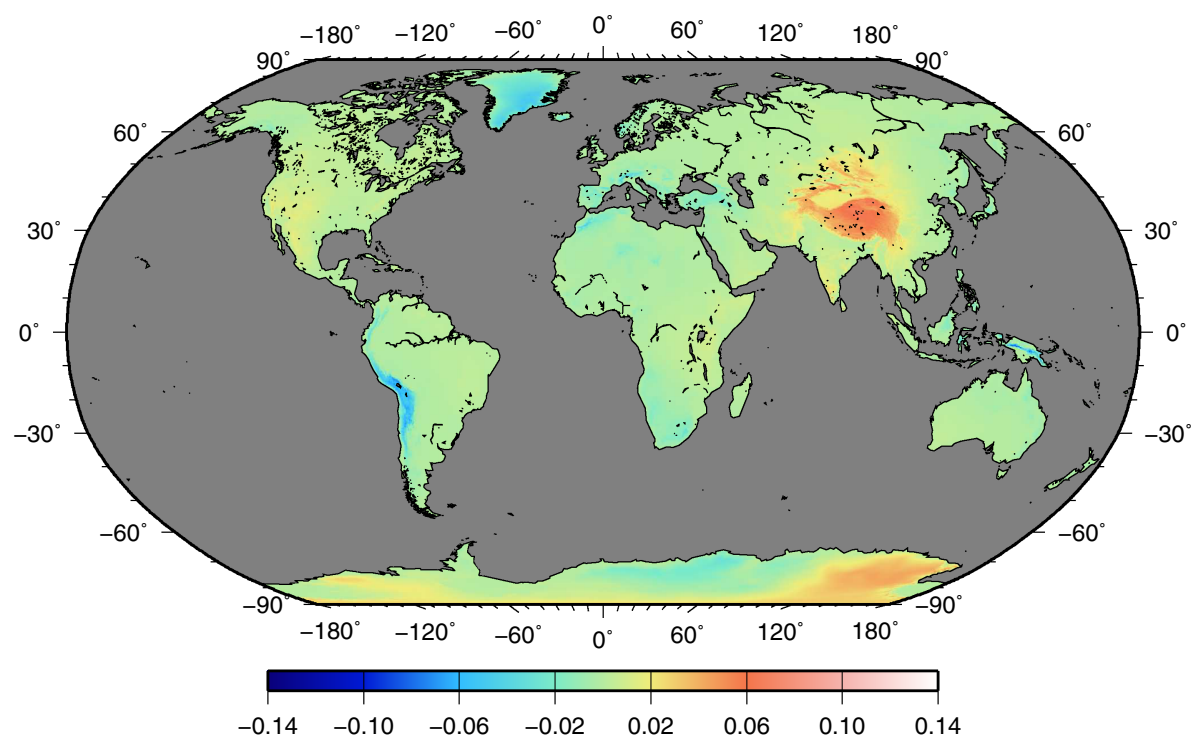

Fig. $1 K_{\gamma}$ (meters) estimated using the 1 arc min global grids of the ETOPO1 global topography (Amante and Eakins 2009) and EGM2008 height anomalies (Pavlis et al. 2012) 


\subsection{Practical implementation of the topographic effects}

Just like $A^{\mathrm{T}}$ the topographic potential $V_{g}^{\mathrm{T}}$ (on the geoid) and $V_{P}^{\mathrm{T}}$ (at the surface point $P$ ) which is needed for the indirect effect $K_{\text {ind }}$ can be derived from a digital elevation model (DEM) by numerical integration.

Sjöberg (2010) proposes to use planar approximation mainly for two reasons: First, the classic definition of the refined Bouguer gravity anomaly incorporates a planar approximation, and second, the (primary) indirect effect of the Bouguer plate vanishes. Therefore, only the corresponding terrain potential $d V_{g}^{\mathrm{T}}$ and $d V_{P}^{\mathrm{T}}$, respectively, needs to be considered from this perspective. In this context it is also argued that the difference of the huge potential values $V^{\mathrm{T}}$ which are infinite for the Bouguer plate should be avoided in numerical computations.

However, Vaníček et al. (2004) pointed out that the classic expression of the complete Bouguer gravity anomaly w.r.t. to an infinite plate is not harmonic in a strict sense and, as such, not appropriate for the task of downward continuation - as it is expressed by the term $K_{\Delta \delta g}$. In contrast, the approach presented here is not limited to the planar case as $A^{\mathrm{T}}$ is not necessarily confined to the classic (planar) definition of the Bouguer reduction. Instead, we formulate the decomposition of the topography into a regular body and a corresponding residual (irregular) terrain for the spherical case. This is of interest considering that, firstly, DEM are usually given in geodetic coordinates (i.e., with good approximation, spherical coordinates) and, secondly, discretization bodies such as tesseroids are increasingly being used having much advantage for the computation of topographic effects in spherical approximation (e.g. Heck and Seitz 2007; Grombein et al. 2013).

In the following, we only consider the regional evaluation of the topography using a limited integration radius. We are aware that on modern computers topographic effects can be computed globally, either by integration in the space domain (e.g. Grombein et al. 2014) or in terms of spherical harmonics (e.g. Hirt 2013). However, such a global approach may not be feasible in a regional study, in particular if ultra-high resolution topography grids are available (e.g., a $25 \mathrm{~m}$, or even a $10 \mathrm{~m}$ DEM, as it is the case in Germany).

Considering the term $K_{\text {ind }}$, the terrain potential then needs to be complemented by a further correction which takes into account the indirect effect of a spherical cap. Let the spherical cap be bounded by the radii $r_{1}=R$ and $r_{2}=R+H_{P}$, where again $H_{P}$ is the topographic height. We consider the radius $\psi$ of the spherical cap to be consistent with the maximum integration radius used for the topographic attraction $A^{\mathrm{T}}$ and the terrain potential $d V$. Note that the indirect effect $V_{g}-V_{P}$ discussed here is different from the so-called "topographic bias" which is independent from the cap size (Sjöberg 2007). Therefore, the separation of the topographic potential difference into the spherical cap part and the terrain part reads

$$
V_{g}^{\mathrm{T}}(\psi)-V_{P}^{\mathrm{T}}(\psi)=V_{g}^{\mathrm{cap}}(\psi)-V_{P}^{\mathrm{cap}}(\psi)+d V_{g}^{\mathrm{T}, \mathrm{sph}}(\psi)-d V_{P}^{\mathrm{T}, \mathrm{sph}}(\psi) .
$$

The $d V^{\mathrm{T}, \text { sph }}$ denote the respective residual terrain potential w.r.t. the spherical cap. The $V^{\text {cap }}$ themselves may take large values, yet they are finite and can be computed analytically. The strict formula (Heck and Seitz 2007, Eq. 52) contains rather complicated expressions. However, taking a numerical approach it was found that the thus obtained difference $V_{g}^{\text {cap }}-V_{P}^{\text {cap }}$ is directly related to the corresponding potential difference for the spherical shell (i.e., the special case where $\psi=\pi$ ). As the overlapping red lines and the black dotted line in Fig. 2 show, it holds 


$$
\frac{V_{g}^{\text {cap }}(\psi)-V_{P}^{\text {cap }}(\psi)}{V_{g}^{\text {shell }}-V_{P}^{\text {shell }}}=\sin \frac{\psi}{2}
$$

The difference of the topographic potential $V^{\text {shell }}$ between the upper and the lower bound is then easy to compute:

$$
V_{g}^{\text {shell }}-V_{P}^{\text {shell }}=\frac{2}{3} \pi G \rho H_{P}^{2}\left(1+\frac{2 R}{R+H_{P}}\right),
$$

where $G$ is the gravitational constant, $\rho$ is a constant density and $R$ is the mean Earth radius. Finally, one obtains

$$
V_{g}^{\mathrm{cap}}(\psi)-V_{P}^{\mathrm{cap}}(\psi)=\frac{2}{3} \pi G \rho H_{P}^{2}\left(1+\frac{2 R}{R+H_{P}}\right) \cdot \sin \frac{\psi}{2}
$$

for the indirect effect of the spherical cap of radius $\psi$. As an example, assuming $\psi=3^{\circ}$ and a density of $\rho=2670 \mathrm{~kg} \mathrm{~m}^{-3}$, the contribution of the spherical cap yields about $3 \mathrm{~mm}, 2.7$ and $23 \mathrm{~cm}$ for surface heights of 1000,3000 and $9000 \mathrm{~m}$, respectively. Alternatively, instead of taking the detour by separating spherical cap and spherical terrain, the complete $V_{g}^{\mathrm{T}}-V_{P}^{\mathrm{T}}$ in the spherical domain may also be directly computed from the DEM.

\subsection{Discussion of additional density contrasts}

The simple relation Eq. 20 is only valid if $P$ is located on the surface of a spherical cap of uniform density. However, this is not anymore the case already if the spherical cap consists of layers of different density. Such multiple density interfaces are formed by, e.g., the

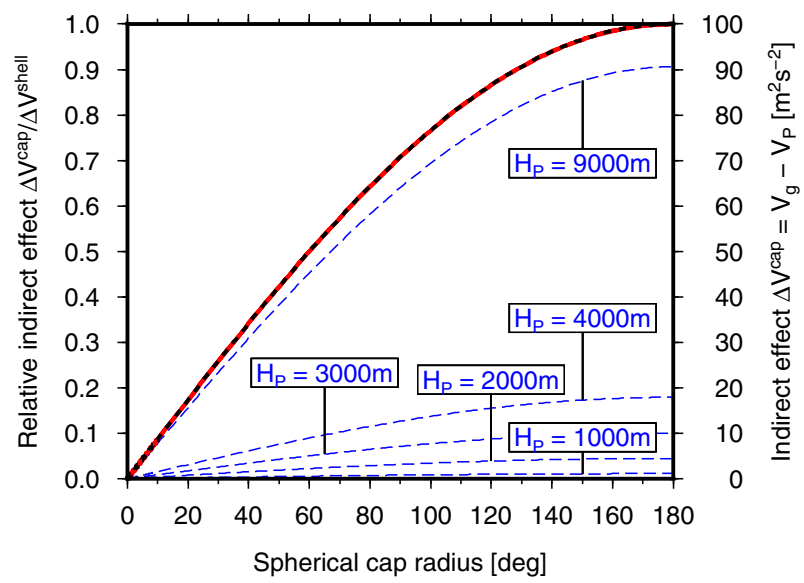

Fig. 2 Indirect effect $V_{g}-V_{P}$ of a spherical cap according to the strict formula (Heck and Seitz (2007), Eq. 52) (dashed blue lines) and relative indirect effect w.r.t. to a spherical shell according to the lefthand side of Eq. 18 (overlapping solid red lines), depending on the cap radius $\psi$ and the altitude of the computation point $H_{P}$. The spherical cap is bounded by the radii $R$ and $R+H_{\text {cap. }} P$ is located on the upper boundary $\left(H_{P}=H_{\text {cap }}\right)$ and the density of rock is assumed $\left(\rho=2670 \mathrm{~kg} \mathrm{~m}^{-3}\right)$. The dotted black curve denotes the analytical function $\sin \frac{\psi}{2}$ according to the right-hand side of Eq. 18 
subglacial bedrock topography in ice-covered areas (e.g., Greenland and Patagonia). Fig. 3 shows a simulation for such a scenario.

In this case, the spherical cap is constructed by superimposing two layers of bedrock and ice, respectively. The height of the subglacial topography is taken as $1000 \mathrm{~m}$, therefore the underlying bedrock layer has a thickness of $1000 \mathrm{~m}$. The point $P$ is located on the surface of the ice layer, and the subscript $g$ refers to the geoid $(H=0)$ which is equivalent to the bottom of the bedrock layer. For simplicity, we set the density of ice to zero, so only the density contrast of bedrock and ice needs to be evaluated. Hence, the point $P$ is now located as in "free air" with respect to the bedrock surface. The indirect effect $V_{g}^{\text {cap }}-V_{P}^{\text {cap }}$ (dashed blue lines) was again computed by strictly evaluating the cumbersome analytical formula (e.g. Heck and Seitz 2007, Eq. 52). The solid red lines indicate the relative effect of the spherical cap with respect to the corresponding spherical shell. It becomes obvious that in case of multiple density layers the indirect effect of the residual density layer increases much faster already at small cap sizes which should be considered carefully when applying Eq. 17.

\section{Numerical calculations with model data}

The Himalaya mountains feature the highest and among the roughest topography on the Earth, so that the largest figures for the contributors to $N-\zeta$ can be expected there. The long-wavelength (commensurate to the resolution of a GOCE model) pattern of the GQS in this region has already been inspected by Bagherbandi and Tenzer (2013), but so far only based on the approximation (Eq. 4) by means of the Bouguer plate reduction. Therefore,

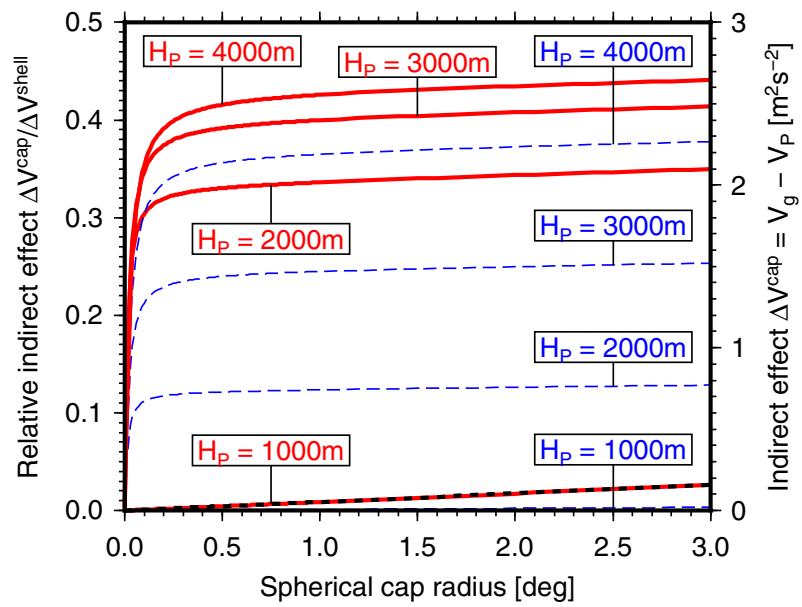

Fig. 3 Indirect effect $V_{g}-V_{P}$ of a spherical cap (dashed blue lines) and relative indirect effect w.r.t. to a spherical shell (solid red lines), depending on the cap radius $\psi$ and the altitude of computation point $H_{P}$ (see caption of Fig. 2 for further explanations). Similarly to Fig. 2, the spherical cap is bounded by the radii $R$ and $R+H_{\text {cap }}$ with $H_{\text {cap }}=1000 \mathrm{~m}$. Again, the dotted black curve denotes the analytical function $\sin \frac{\psi}{2}$. However, differently from Fig. 2 the altitude $H_{P}$ now varies $\left(H_{P} \geq H_{\text {cap }}\right)$ and the density of the cap is now $\Delta \rho=\rho_{\text {bedrock }}-\rho_{\text {ice }}=1753 \mathrm{~kg} \mathrm{~m}^{-3}$. Thus, this scenario resembles the additional density contrast of a subglacial bedrock topography: The computation point $P$ located at the ice surface of varying thickness is in "free air" with respect to the spherical cap of residual density $\Delta \rho$, so that Eq. 18 is no longer valid 
the Himalaya and the adjacent region $\left(60^{\circ}-105^{\circ} \mathrm{E}, 15^{\circ}-45^{\circ} \mathrm{N}\right)$ provide a suitable test area to estimate the magnitude and spatial pattern of the individual corrections. Furthermore, we want to study the effect of the integration radius on the results. Therefore, we intend our numerical study to be understood rather as a simulation based on "error-free data".

The height anomaly and gravity anomaly grids provided with EGM2008 (Pavlis et al. 2012, 2013) and the 1 arc min DEM of ETOPO1 (Amante and Eakins 2009) served as realistic data for this simulation. Contrary to using spherical caps as described in Sect. 2.3, the topographic effects $A^{\mathrm{T}}$ and $V_{g}^{\mathrm{T}}-V_{P}^{\mathrm{T}}$ were directly computed by means of prism integration with respect to a reference sphere and applying different integration radii (20, $100,300,500 \mathrm{~km})$. To this end, the TC program of the GRAVSOFT software package (Forsberg and Tscherning 2008) was modified to allow computation points at the lower bound of the topography. Regarding term $K_{\Delta \delta g}$ the approximation according to Eq. 16 was introduced. In order to estimate the vertical gradient of the non-topographic gravity disturbance $\left(\frac{\partial}{\partial h} \delta g^{\mathrm{nt}}\right)_{P}$ we made use of the GRAVSOFT program GEOFOUR (Fourier transform in planar approximation).

EGM2008 provides a nominal spectral resolution around $18 \mathrm{~km}$. Thus, the non-topographic gravity disturbance $\delta g^{\mathrm{nt}}=\delta g-A^{\mathrm{T}}$ needs to be low-pass filtered to ensure consistency between the gravity model and the DEM. Otherwise, the omission error of EGM2008 will appear as high-frequency noise in $K_{\delta g}$ and, thus, in $K_{\Delta \delta g}$. We found that a simple $20 \mathrm{~km}$ boxcar filter applied to $\delta g^{\mathrm{nt}}$ and a $20 \mathrm{~km}$ Wiener filter in the estimation of the gravity gradient provided reasonably smooth results. Hence, the finest topographic structures of the total $N-\zeta$ effect cannot be resolved in our numerical experiment. However, the study of differences alone, only due to changes of the integration radius, enables useful insights related to the indirect effect $K_{\text {ind }}$ also at the full spectral resolution of the DEM. To this end, in the following we will refer to the difference of two effects $(*)$ associated with different underlying representations of topography $a$ and $b$ by the notation ${ }_{\mathrm{a}}^{\mathrm{b}} \Delta(*)={ }^{\mathrm{b}}(*)-{ }^{\mathrm{a}}(*)$, where numbers stand for the integration radius in $\mathrm{km}$ in the spherical case and plate $(*)$ denotes the infinite Bouguer plate without terrain corrections.

However, we did not finally filter the results of the individual $K$ terms itself. The only purpose of the abovementioned filter is to ensure that the simulated $\delta g^{\text {nt }}$ data are reasonably smooth, as it could be expected already without filtering if real terrestrial gravity data were used. However, even then $K_{\delta g}$ and $K_{\text {ind }}$ would anyway show a different spectral pattern than $\delta g^{\text {nt }}$. On the one hand, $K_{\delta g}$ depends on the smooth $\delta g^{\text {nt }}$ and the possibly rough topographic height. Thus, over rough terrain $K_{\delta g}$ is likely to contain signal at higher frequencies compared to $\delta g^{\text {nt }}$ itself! On the other hand, if $\delta g^{\text {nt }}$ is considered filtered but the topographic attraction $A^{\mathrm{T}}$ (Eq. 10) itself is not, the effective resolution of $K_{\text {ind }}$ is then also only related to that of the DEM.

The EGM2008 height anomalies served to estimate $K_{\gamma}$, in the same manner as demonstrated in Fig. 1 for the whole Earth, and to convert the grid of gravity anomalies to gravity disturbances, which were resampled to the 1 arc min grid of the DEM. The nontopographic gravity disturbance was then computed as $\delta g^{\mathrm{nt}}=\delta g^{\mathrm{EGM} 2008}-A^{\mathrm{T}}$ and subsequently filtered as explained above. From this product, the vertical gradients were estimated. Finally, $K_{\delta g}, K_{\Delta \delta g}$ and $K_{\text {ind }}$ were computed according to Eqs. 15 b, 16 and 15 d, respectively. The results related to a $500 \mathrm{~km}$ integration radius are illustrated in Fig. 4 and Table 1.

In our example $K_{\gamma}$ takes positive values at the order of some centimeters and is rather weakly (in contrast to the terms $K_{\delta g}$ and $K_{\text {ind }}$ ) correlated with the topography. According to 
the regional behavior of the non-topographic gravity disturbance $\delta g^{\text {nt }}$ the term $K_{\delta g}$, and thus $N-\zeta$, is mainly negative and at the order of 2-3 $\mathrm{m}$ in the highland of Tibet which is in agreement with the figures as reported by e.g. Flury and Rummel (2009). As it is well known, $K_{\delta g}$ is strongly, i.e., almost linearly, correlated with topography. Its maxima coincide with the largest elevations of topography. In contrast, a comparison with a map of slopes derived from the DEM (not shown here) confirmed that the strongest signal in $K_{\text {ind }}$ appears either along mountain ridges (positive values) or along the adjacent steep slopes of deep and narrow valleys (negative values), as already discussed by Flury and Rummel

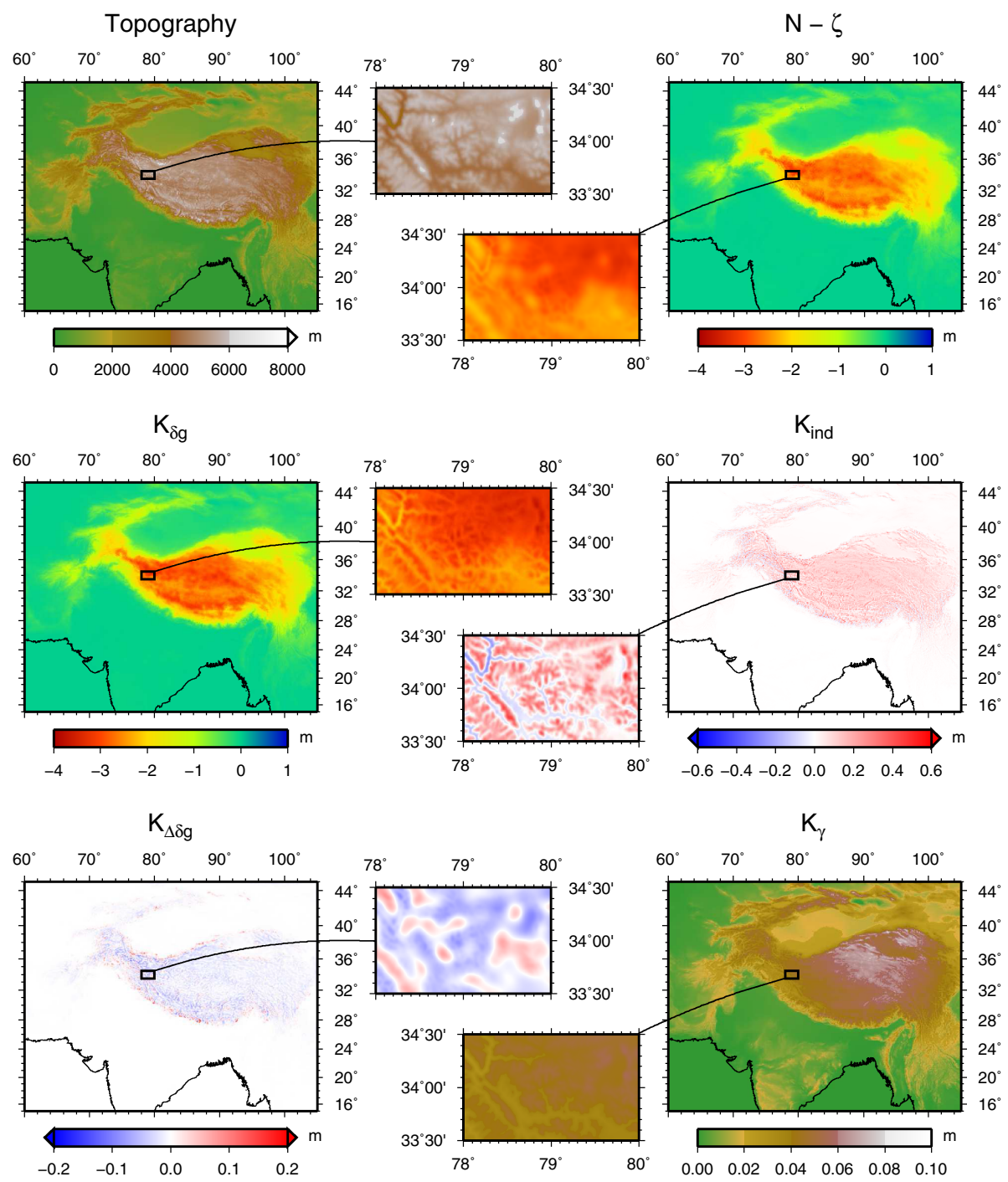

Fig. 4 ETOPO1 global 1 arc min topography, simulated $N-\zeta$ and constituents displayed for the whole Himalaya test area and for a zoom-in in the highlands of Tibet. Spherical representation of the topography with $500 \mathrm{~km}$ integration radius is used 
Table 1 Statistics of simulated $N-\zeta$ and its constituents for a spherical representation of the topography with $500 \mathrm{~km}$ integration radius

\begin{tabular}{|c|c|c|c|c|c|c|c|c|}
\hline & \multicolumn{4}{|c|}{ Himalaya test area } & \multicolumn{4}{|c|}{ Tibet zoom-in area } \\
\hline & Mean & SD & Min. & Max. & Mean & SD & Min. & Max. \\
\hline$K_{\gamma}$ & +0.018 & 0.019 & +0.000 & +0.090 & +0.043 & 0.005 & +0.028 & +0.057 \\
\hline$K_{\delta g}$ & -0.543 & 0.867 & -3.768 & +0.044 & -2.863 & 0.322 & -3.768 & -1.964 \\
\hline$K_{\Delta \delta g}$ & -0.002 & 0.010 & -0.133 & +0.360 & -0.018 & 0.033 & -0.095 & +0.086 \\
\hline$K_{\text {ind }}$ & +0.020 & 0.051 & -0.324 & +1.538 & +0.101 & 0.126 & -0.318 & +0.550 \\
\hline$N-\zeta$ & -0.506 & 0.821 & -3.387 & +1.012 & -2.738 & 0.300 & -3.387 & -2.123 \\
\hline
\end{tabular}

The test areas refer to Fig. 4

Units are in meters

(2009). However, the indirect effect is close to zero over high plateaus. In this way, $K_{\text {ind }}$ partly compensates for the short-wavelength signal in $K_{\delta g}$ resulting in a notably smoothed and slightly decorrelated total effect $N-\zeta$. Finally, the vertical change of $\delta g^{\text {nt }}$ along the plumbline yields estimates of $K_{\Delta \delta g}$ up to the order of some decimeters in rugged terrain. At the short scales $K_{\Delta \delta g}$ is as well partly correlated with topography as it depends on elevation. However, it also exhibits a characteristic pattern on a coarser scale. All over the highland of Tibet it is mainly negative, whereas the steep boundaries with the adjacent lowlands are dominated by positive values.

Looking at Fig. 5 and Table 2, it becomes clear that both ${ }_{\mathrm{a}}^{\mathrm{b}} \Delta K_{\text {ind }}$ and ${ }_{\mathrm{a}}^{\mathrm{b}} \Delta(N-\zeta)$ converge toward zero for increasing integration radii. Already $20 \mathrm{~km}$ is sufficient in low and flat terrain. An integration radius of $100 \mathrm{~km}$ covers the regional signal of flat basins and, down to the decimeter, most of the terrain effects adjacent to steep mountains. Finally, the omission error ${ }_{300}^{500} \Delta(N-\zeta)$ does not exceed $1.4 \mathrm{~cm}$ for the given DEM resolution of 1 arc min. The contribution of $K_{\Delta \delta g}$ appears to be almost independent from the topographic model. Certainly, based on a rather crude global DEM and gravity model our simulation omits the local ultra-short wavelength signal in areas of very rough terrain. In this context, Flury and Rummel (2009) discussed that a DEM resolution of $1 \mathrm{~km}$ may result in errors of up to $7 \mathrm{~cm}$. However, the main characteristic behavior of $N-\zeta$ in rough terrain is resolved in our experiment.

In this context, we also consider the simple plate approximation plate $A^{\mathrm{T}}=2 \pi G \rho H_{P}$. As already mentioned, the indirect effect ${ }^{\text {plate }} K_{\text {ind }}$ of an infinite plate vanishes. Thus, the question is whether $N-\zeta$ could be computed strictly without any terrain reduction. Following the ideas of Sjöberg (2007) this scenario may be imagined as a situation where the residual terrain w.r.t. the infinite plate is "hovering" and has a borehole of infinitely small radius in $P$ so that the plumbline is theoretically in "free air." However, in this case the first-order approximation underlying Eq. 16 will not suit the actual gravity gradient according to Eq. 14, which in this hypothetical situation still contains the unmodelled effect of the terrain. This is confirmed by examining Fig. 5 and Table 2. In other words, in case that $A^{\mathrm{T}}$ is constructed of the infinite Bouguer plate without any terrain correction, the avoided $K_{\text {ind }}$ is just algebraically shifted to the strict $K_{\Delta \delta g}$ according to Eq. 15c [compare also Flury and Rummel (2009), Eq. 19 and Sects. 4 and 6]. 


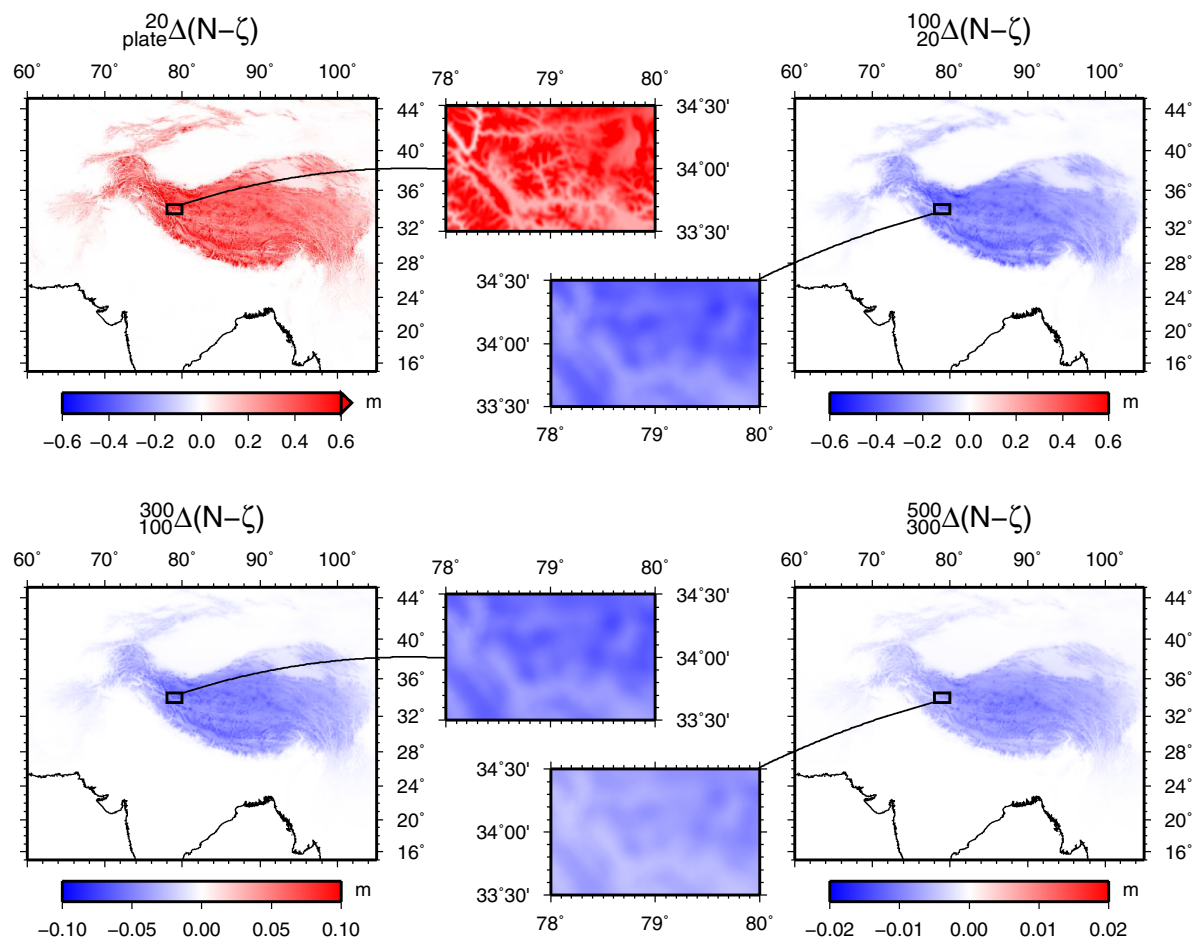

Fig. 5 Differences of simulated $N-\zeta$ for selected representations of topography: infinite plate versus spherical representation with different integration radii, displayed for the whole Himalaya test area and for a zoom-in in the highlands of Tibet

\section{Conclusions}

A strict formulation of the GQS term is deduced based on the non-topographic gravity disturbance. With respect to the topographic reduction the approach is general, i.e., it is not confined to the classic planar Bouguer reduction. As Sjöberg (2012) points out, the approach may be applied for any arbitrary topographic reduction model, e.g., using isostatic anomalies. The only requirements (see Eq. 15a) are that, firstly, the (surface) height anomaly $\zeta$ is a-priori known, secondly, the representation of the topography is the same for $A^{\mathrm{T}}$ and $V^{\mathrm{T}}$, and, thirdly, the terrain potential $d V^{\mathrm{T}}$ in $K_{\text {ind }}$ is properly complemented by the non-vanishing indirect effect of a spherical cap (see Eq. 20) if a spherical representation of the topography is to be used and $V^{\mathrm{T}}$ is not evaluated directly.

This formula is not restricted to the geoid. It may be used to evaluate the downwardcontinuation of $\zeta$, or synonymously of $T$, inside the topography to any arbitrary level (or, more general, surface) $H_{P^{\prime}}$. For example, it has been adapted to infer the equilibrium surface of the subglacial Lake Vostok, Antarctica (Schwabe et al. 2014). Given that the topography in $A^{\mathrm{T}}$ and $V^{\mathrm{T}}$ is defined accordingly, one only needs to replace the geoidrelated quantities $(*)_{g}$ by $(*)_{P^{\prime}}, \gamma_{0}$ by $\gamma_{Q^{\prime}}$ (related to to the telluroid point $Q^{\prime}$ associated with $P^{\prime}$ ) and, finally, $H_{P}$ by $H_{P}-H_{P^{\prime}}$. In case that $H_{P^{\prime}}$ is not constant also the respective terrain potential related to the lower bound of the topography needs to be considered. 
Table 2 Statistics of differences of simulated $N-\zeta$ and its constituents for selected representations of topography: infinite plate versus spherical representation and different integration radii

\begin{tabular}{|c|c|c|c|c|c|c|c|c|}
\hline & \multicolumn{4}{|c|}{ Himalaya test area } & \multicolumn{4}{|c|}{ Tibet zoom-in area } \\
\hline & Mean & SD & Min. & Max. & Mean & SD & Min. & Max. \\
\hline \multicolumn{9}{|l|}{ Differences $\Delta K_{\delta g}$} \\
\hline${ }_{\text {plate }}^{20} \Delta K_{\delta g}$ & +0.066 & 0.122 & +0.000 & +0.994 & +0.427 & 0.084 & +0.219 & +0.626 \\
\hline${ }_{20}^{100} \Delta K_{\delta g}$ & -0.051 & 0.098 & -0.629 & +0.008 & -0.336 & 0.071 & -0.506 & -0.174 \\
\hline${ }_{100}^{300} \Delta K_{\delta g}$ & -0.016 & 0.028 & -0.139 & +0.000 & -0.098 & 0.016 & -0.135 & -0.057 \\
\hline${ }_{300}^{500} \Delta K_{\delta g}$ & -0.009 & 0.014 & -0.067 & +0.000 & -0.038 & 0.005 & -0.053 & -0.023 \\
\hline \multicolumn{9}{|l|}{ Differences $\Delta K_{\Delta \delta g}$} \\
\hline plate ${ }^{20} \Delta K_{\Delta \delta g}$ & +0.001 & 0.003 & -0.015 & +0.119 & +0.005 & 0.008 & -0.013 & +0.027 \\
\hline${ }_{20}^{100} \Delta K_{\Delta \delta g}$ & -0.001 & 0.002 & -0.068 & +0.020 & -0.004 & 0.006 & -0.020 & +0.009 \\
\hline${ }_{100}^{300} \Delta K_{\Delta \delta g}$ & +0.000 & 0.000 & -0.008 & +0.003 & -0.001 & 0.001 & -0.004 & +0.001 \\
\hline${ }_{300}^{500} \Delta K_{\Delta \delta g}$ & +0.000 & 0.000 & -0.001 & 0.000 & -0.000 & 0.000 & -0.001 & +0.000 \\
\hline \multicolumn{9}{|l|}{ Differences $\Delta K_{\text {ind }}$} \\
\hline${ }_{\text {plate }}^{20} \Delta K_{\text {ind }}$ & +0.001 & 0.029 & -0.331 & +1.115 & +0.003 & 0.091 & -0.310 & +0.346 \\
\hline${ }_{20}^{100} \Delta K_{\text {ind }}$ & +0.004 & 0.011 & -0.060 & +0.311 & +0.020 & 0.029 & -0.052 & +0.103 \\
\hline${ }_{100}^{300} \Delta K_{\text {ind }}$ & +0.008 & 0.014 & +0.000 & +0.086 & +0.047 & 0.009 & +0.020 & +0.073 \\
\hline${ }_{300}^{500} \Delta K_{\text {ind }}$ & +0.007 & 0.012 & +0.000 & +0.057 & +0.031 & 0.004 & +0.018 & +0.044 \\
\hline \multicolumn{9}{|c|}{ Differences $\Delta(N-\zeta)$} \\
\hline${ }_{\text {plate }}^{20} \Delta(N-\zeta)$ & +0.067 & 0.132 & -0.066 & +2.170 & +0.435 & 0.157 & +0.030 & +0.940 \\
\hline${ }_{20}^{100} \Delta(N-\zeta)$ & -0.048 & 0.092 & -0.504 & +0.001 & -0.320 & 0.054 & -0.448 & -0.196 \\
\hline${ }_{100}^{300} \Delta(N-\zeta)$ & -0.008 & 0.015 & -0.072 & +0.000 & -0.052 & 0.008 & -0.071 & -0.034 \\
\hline${ }_{300}^{500} \Delta(N-\zeta)$ & -0.001 & 0.002 & -0.013 & +0.000 & -0.007 & 0.001 & -0.010 & -0.004 \\
\hline${ }_{\text {plate }}^{500} \Delta(N-\zeta)$ & +0.009 & 0.039 & -0.313 & +1.516 & +0.048 & 0.100 & -0.229 & +0.550 \\
\hline
\end{tabular}

The test areas refer to Fig. 5

Units are in meters

Our numerical simulation for the Himalayan region confirms the anticipated behavior of $N-\zeta$ as described by Flury and Rummel (2009). Whereas in lowlands and flat basins the well-known approximation by means of the Bouguer anomaly (Eq. 4) gives results 
accurate to the centimeter level, the effect of rough terrain is not limited to the flanks and valleys in the direct vicinity of large peaks. The total (direct and indirect) contribution of topography may exceed $1 \mathrm{~cm}$ even in a far zone up to $500 \mathrm{~km}$ (see the omission error ${ }_{300}^{500} \Delta(N-\zeta)$ displayed in Fig. 5 and Table 2) which should be considered. Future simulations may focus on the magnitude and spatial pattern of short-scale variations of $K_{\Delta \delta g}$, provided a high-resolution gravity model is available that is spectrally consistent with the topographic effects modeled by means of the DEM.

\section{References}

Amante C, Eakins BW (2009) ETOPO1 1 Arc-minute global relief model: procedures, data sources and analysis. NOAA Technical memorandum NESDIS NGDC-24, 19 pp, March 2009

Bagherbandi M, Tenzer R (2013) Geoid-to-quasigeoid separation computed using the GRACE/GOCE global geopotential model GOCO02S - a case study of Himalayas and Tibet. Terr, Atmos Ocean Sci 24(1):59-68. doi:10.3319/TAO.2012.09.17.02(TT)

Barthelmes F (2009) Definition of functionals of the geopotential and their calculation from spherical harmonic models: theory and formulas used by the calculation service of the International Centre for Global Earth Models (ICGEM), http://icgem.gfz-potsdam.de. Tech. rep., doi:10.2312/GFZ.b103-09026

Flury J, Rummel R (2009) On the geoid-quasigeoid separation in mountain areas. J Geod 83(9):829-847. doi:10.1007/s00190-009-0302-9

Flury J, Rummel R (2011) On the computation of the geoid-quasigeoid separation. J Geod 85(3):185-186. doi:10.1007/s00190-011-0447-1

Forsberg R, Tscherning C (2008) An overview manual for the GRAVSOFT Geodetic Gravity Field Modelling Programs. 2nd edn. Contract report for JUPEM. http://cct.gfy.ku.dk/publ_cct/cct1792 (last Accessed: 02 July 2013)

Grombein T, Seitz K, Heck B (2013) Optimized formulas for the gravitational field of a tesseroid. J Geod 87(7):645-660. doi:10.1007/s00190-013-0636-1

Grombein T, Luo X, Seitz K, Heck B (2014) A wavelet-based assessment of topographic-isostatic reductions for GOCE gravity gradients. Surv Geophys 35(4):959-982. doi:10.1007/s10712-014-9283-1

Heck B, Seitz K (2007) A comparison of the tesseroid, prism and point-mass approaches for mass reductions in gravity field modelling. J Geod 81(2):121-136. doi:10.1007/s00190-006-0094-0

Heiskanen W, Moritz H (1967) Physical Geodesy. Freeman, San Francisco London

Hirt C (2013) RTM Gravity forward-modeling using topography/bathymetry data to improve high-degree global geopotential models in the coastal zone. Mar Geod 36:183-202. doi:10.1080/01490419.2013. 779334

Pavlis N, Holmes S, Kenyon S, Factor J (2012) The development and evaluation of the Earth gravitational model 2008 (EGM2008). J Geophys Res 117(B4):406. doi:10.1029/2011JB008916

Pavlis NK, Holmes SA, Kenyon SC, Factor JK (2013) Correction to "The development and evaluation of the Earth gravitational model 2008 (EGM2008)". J Geophys Res: Solid Earth 118(5):2633-2633. doi:10.1002/jgrb.50167

Rapp R (1997) Use of potential coefficient models for geoid undulation determinations using a spherical harmonic representation of the height anomaly/geoid undulation difference. J Geod 71(5):282-289. doi:10.1007/s001900050096

Schwabe J, Ewert H, Scheinert M, Dietrich R (2014) Regional geoid modeling in the area of subglacial Lake Vostok, Antarctica. J Geodyn 75:9-21. doi:10.1016/j.jog.2013.12.002

Sjöberg L (1995) On the quasigeoid to geoid separation. Manuscripta Geod 20(3):182-192

Sjöberg L (2006) A refined conversion from normal height to orthometric height. Studia Geophys et Geod 50(4):595-606. doi:10.1007/s11200-006-0037-5

Sjöberg L (2007) The topographic bias by analytical continuation in physical geodesy. J Geodesy 81(5):345-350. doi:10.1007/s00190-006-0112-2

Sjöberg L (2010) A strict formula for geoid-to-quasigeoid separation. J Geod 84(11):699-702. doi:10.1007/ s00190-010-0407-1

Sjöberg L (2012) The geoid-to-quasigeoid difference using an arbitrary gravity reduction model. Studia Geophys et Geod 56(4):929-933. doi:10.1007/s11200-011-9037-1

Tenzer R, Vaníček P, Santos M, Featherstone W, Kuhn M (2005) The rigorous determination of orthometric heights. J Geod 79(1-3):82-92. doi:10.1007/s00190-005-0445-2 
Tenzer R, Novák P, Moore P, Kuhn M, Vaníček P (2006) Explicit formula for the geoid-quasigeoid separation. Studia Geophys et Geod 50(4):607-618. doi:10.1007/s11200-006-0038-4

Vaníček P, Tenzer R, Sjöberg LE, Martinec Z, Featherstone WE (2004) New views of the spherical Bouguer gravity anomaly. Geophys J Int 159(2):460-472. doi:10.1111/j.1365-246X.2004.02435.x

Vaníček P, Kingdon R, Santos M (2012) Geoid versus quasigeoid: a case of physics versus geometry. Contrib Geophys Geod 42(1):101-118. doi:10.2478/v10126-012-0004-9 